\title{
Genomic and gene regulatory signatures of cryptozoic adaptation: Loss of blue sensitive photoreceptors through expansion of long wavelength-opsin expression in the red flour beetle Tribolium castaneum
}

\author{
Magdalena Jackowska ${ }^{1}$, Riyue Bao ${ }^{1}$, Zhenyi Liu ${ }^{2}$, Elizabeth C McDonald ${ }^{3}$, \\ Tiffany A Cook ${ }^{3}$ and Markus Friedrich*1,4
}

Address: ${ }^{1}$ Department of Biological Sciences, Wayne State University, Detroit, MI 48202 USA, ${ }^{2}$ Department of Molecular Biology and Pharmacology, Washington University in St Louis School of Medicine, 3600 Cancer Research Building, St. Louis, MO 63110 USA, ${ }^{3}$ Division of Developmental Biology and Department of Pediatric Ophthalmology, Cincinnati Children's Hospital Medical Center, Cincinnati OH 45229 USA and ${ }^{4}$ Department of Anatomy and Cell Biology, Wayne State University School of Medicine, Detroit, MI 48201 USA

Email: Magdalena Jackowska - magdallena_jackowska@yahoo.com; Riyue Bao - ry_bao@yahoo.com; Zhenyi Liu - liuzhenyi@wustl.edu; Elizabeth C McDonald - elizabeth.mcdonald@cchmc.org; Tiffany A Cook - tiffany.cook@cchmc.org;

Markus Friedrich* - mf@biology.biosci.wayne.edu

* Corresponding author

Published: 21 December 2007

Frontiers in Zoology 2007, 4:24 doi:10.1 186/1742-9994-4-24
Received: 19 September 2007

Accepted: 21 December 2007

This article is available from: http://www.frontiersinzoology.com/content/4/I/24

(C) 2007 Jackowska et al; licensee BioMed Central Ltd.

This is an Open Access article distributed under the terms of the Creative Commons Attribution License (http://creativecommons.org/licenses/by/2.0), which permits unrestricted use, distribution, and reproduction in any medium, provided the original work is properly cited.

\begin{abstract}
Background: Recent genome sequence analysis in the red flour beetle Tribolium castaneum indicated that this highly crepuscular animal encodes only two single opsin paralogs: a UV-opsin and a long wavelength (LW)-opsin; however, these animals do not encode a blue (B)-opsin as most other insects. Here, we studied the spatial regulation of the Tribolium single LW- and UV-opsin gene paralogs in comparison to that of the five opsin paralogs in the retina of Drosophila melanogaster.

Results: In situ hybridization analysis reveals that the Tribolium retina, in contrast with other insect retinas, constitutes a homogenous field of ommatidia that have seven LW-opsin expressing photoreceptors and one UV-/LW-opsin co-expressing photoreceptor per eye unit. This pattern is consistent with the loss of photoreceptors sensitive to blue wavelengths. It also identifies Tribolium as the first example of a species in insects that co-expresses two different opsins across the entire retina in violation of the widely observed "one receptor rule" of sensory cells.
\end{abstract}

Conclusion: Broader studies of opsin evolution in darkling beetles and other coleopteran groups have the potential to pinpoint the permissive and adaptive forces that played a role in the evolution of vision in Tribolium castaneum. 


\section{Introduction}

Photon absorption by visual pigments is the primary event in the conversion of light to a neuronal signal in animal photoreceptor cells [1]. Visual pigments or rhodopsins form by an association of retinal-based chromophores with a special class of G-protein-coupled seven transmembrane receptor proteins (GPCRs). These GPCRs are encoded by a diverse group of opsin gene family paralogs. Most insects encode members of three different opsin subfamilies. Ultraviolet (UV) and blue (B) opsins correlate with maximal photoreceptor sensitivities in the 300-400 and $400-500 \mathrm{~nm}$ ranges respectively. Long wavelength (LW) opsin-expressing photoreceptors show sensitivities that range between 480 and $600 \mathrm{~nm}$ [2$4]$. The differential expression of these diverged opsin paralogs in the photoreceptors of individual eye units (ommatidia) within the compound eye is a structural prerequisite for color discrimination by insects. Moreover, differential opsin expression can correlate with cellular specialization in color and brightness detection in insects. In Drosophila, brightness is measured from the input of six peripheral LW-opsin expressing photoreceptors (R1-R6), which project into the first optic neuropil, the lamina. Color vision is facilitated by the central photoreceptors R7 and 8 that express one of the UV-, B-, or LW-opsins and have long axonal projections that synapse into the second optic neuropil, the medulla (Fig. 1) (for review see [4-6]).

The existence of UV-, B- and LW-sensitive photoreceptors in primitive insects implies that trichromatic vision is ancient and highly conserved [2]. Consistent with this, recent genome sequencing projects identified UV-, B- and LW-opsin gene family paralogs in diverse endopterygote insects including silkmoth, mosquito and honeybee [7]. Remarkably, only two opsin gene orthologs representing members of the LW- and UV-opsin groups were detected in the genome of the red flour beetle Tribolium castaneum, suggesting the loss of B-opsin during the evolution of this species $[7,8]$ (Bao, Friedrich, in preparation). Like darkling beetles (Tenebrionidae) in general, the stored grain pest Tribolium castaneum is highly secretive and avoids broad daylight: all postembryonic life cycle stages hide in tunnels driven through the food substrate $[9,10]$. The dramatic increase of chemoreceptor genes, compared to diurnal species like honeybee and Drosophila, suggests a strong adaptive effect of Tribolium's cryptozoic ecology on its genome and neurobiology [8]. Thus, it is conceivable that a relaxation of color discrimination requirements allowed for the loss of B-opsin in Tribolium. However, there are also examples of trichromatic dark active insect species like the tobacco hornworm moth Manduca sexta [11].

The highly regulated spatial expression of opsin paralogs in Drosophila (Fig. 1) and other endopterygote species allows specific predictions regarding the expression of the a

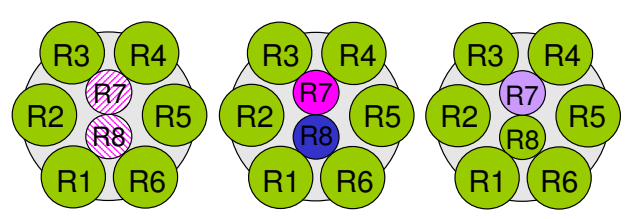

b

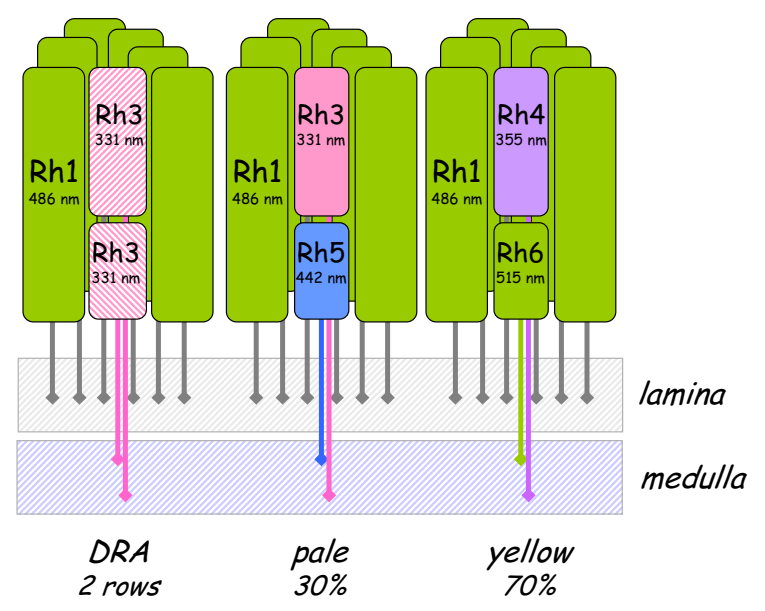

Figure I

Differential opsin expression in Drosophila. Schematic drawings of photoreceptor cell arrangements in the three types of ommatidia that exist in the retina of Drosophila melanogaster, and differ in the choice of differentially expressed opsin paralogs. (a) Cross-section perspective. It should be noted that the R7 and R8 rhabdomeres are represented in the same plane for ease in representation. (b) Sagital section perspective. In pale-type ommatidia, the peripheral photoreceptors RI-R6 express the LW-opsin RhI, the R7 photoreceptor cell expresses the UV-opsin paralog Rh3, and the R8 photoreceptor cell expresses the B-opsin paralog Rh5. Yellow differ from pale ommatidia by expression of the UVopsin paralog Rh4 in the R7 cells, and LW-opsin paralog Rh6 in R8. Dorsal rim area (DRA) ommatidia express Rh3 in both the R7 and R8 cell [12].

remaining two opsin paralogs in Tribolium if B-opsin is missing $[5,12]$. That is, in the absence of B-opsin, the ancestral B-opsin expression niche in a fraction of the central R7 and R8 cells should be replenished by either UVor LW-expression. By examining the expression patterns of the identified LW- and UV-opsin genes in the Tribolium retina, we sought to answer if the spatial regulation of these genes was consistent with a loss of B-opsin, and if so, which specific changes in the regulation of either LW- or UV-opsin expression would be associated with it.

\section{Results}

Each Tribolium ommatidium contains eight photoreceptors, each of which is unambiguously related to one of the photoreceptor subtypes R1-R8 of Drosophila by position, rhabdomere structure and ontogenetic origin [13]. We therefore compared the spatial distribution of Drosophila 
UV- and LW-opsins detected by immunohistochemistry with Tribolium UV- and LW-opsin as detected by in situ hybridization (Fig. 2 and data not shown).

\section{Spatial regulation of the expression of the Tribolium UV opsin gene}

We first investigated the expression of Tribolium UV-opsin. To generate a Tribolium UV-opsin RNA probe for whole mount in situ hybridization, we cloned a 502 bp fragment of the predicted Tribolium UV-opsin gene. In Drosophila, the UV-opsin paralogs Rh3 and Rh4 are stochastically distributed in non-overlapping patterns in all central R7 photoreceptor cells (Fig. 1, Fig 2a and 2b) [14,15]. Similarly, in situ hybridization experiments with an RNA antisense probe against Tribolium UV-opsin in mature pupal and early adult retina detected strong expression in a single photoreceptor cell per ommatidia throughout the entire retinal field (Fig. 2d). Differential interference contrast microscopy at high magnification confirmed that the Tribolium UV-opsin paralog was selectively expressed in all R7-type cells (Fig. 2e). The comparison with Drosophila thus revealed that Tribolium UV-opsin is expressed in a conserved R7-specific manner.

\section{Spatial regulation of the expression of Tribolium $L W$ - opsin gene}

Next we investigated the expression of Tribolium LWopsin. In Drosophila, the peripheral photoreceptors R1-6

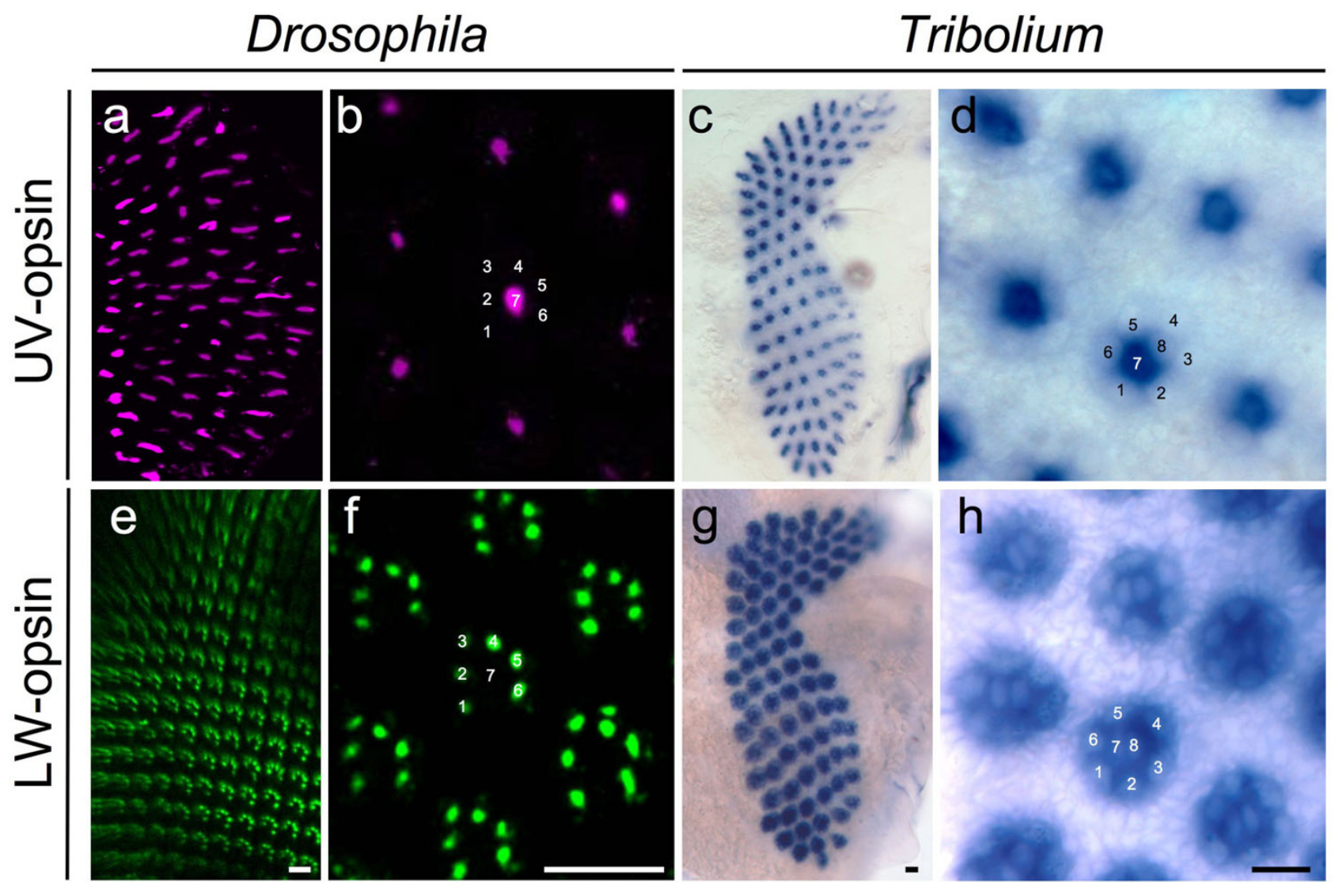

Figure 2

Differential expression of opsin paralogs in Drosophila and Tribolium. (a and b) Digital sections of Drosophila pupal tissue stained with antibodies against UV-sensitive Rh3 and Rh4 opsins. (a) Low magnification overview. (b) High magnification view. Numbers indicate photoreceptor cell subtypes RI-8. (c and d) Tribolium UV-opsin expression detected by in situ hybridization. (c) Low magnification overview of UV-opsin expression throughout entire pupal retina. (d) High magnification view of cell specific expression of Tribolium UV-opsin. Numbers indicate photoreceptor cell subtypes. (e and f) Digital sections of Drosophila pupal tissue stained with antibody against the long wavelength-specific opsin RhI. (e) Low magnification overview. (f) High magnification view. Numbers indicate photoreceptor cell subtypes RI-8. (g and h) Tribolium LW-opsin expression detected by in situ hybridization. (c) Overview of LW-opsin expression throughout entire pupal retina. (d) High magnification of cell specific expression of Tribolium LW-opsin. Numbers indicate photoreceptor cell subtypes. Scale bars correspond to I0 $\mu \mathrm{m}$. 
express the LW-opsin paralog Rh1 (Fig. 2e and 2f), and $70 \%$ of central R8 cells express the LW-opsin Rh6 [16]. The remaining 30\% of R8 cells express the Drosophila Bopsin paralog Rh5 [17,18]. For whole mount in situ hybridization in Tribolium, we cloned a 972 bp long RTPCR fragment that includes the entire predicted Tribolium LW-opsin ORF from head specific Tribolium total RNA. Analysis of the expression of the single Tribolium LWopsin gene revealed uniform transcription in all photoreceptors of the retina (Fig. 2g). Importantly, expression was not only detected in the peripheral photoreceptors, but also in all central R7 and R8 photoreceptors (Fig. 2h). This finding reveals a strongly expanded expression domain of LW-opsin compared to Drosophila, and indicates that R7 cells in Tribolium co-express the LW and UV-opsin-encoding genes.

Like in most insects, ommatidia in the dorsal rim area (DRA) of the Drosophila compound eye exhibit modifications for the detection of polarized light including expression of UV-opsin in both R7 and R8 $[19,20]$. However, no evidence of regional heterogeneity of opsin expression could be detected in the dorsal region of the Tribolium retina (Fig. 2c and 2g), consistent with the lack of morphological evidence of DR ommatidia in this species [13].

\section{Discussion}

Taken together, the new expression data described here reveal a number of unique aspects of the Tribolium compound eye retina: (1) lack of B-opsin encoding photoreceptors, (2) homogeneous expression of a single LWopsin across the entire retina, and (3) co-expression of UV- and LW-opsin in all R7 cells. These findings generate questions regarding the evolutionary mechanisms, forces and pathways that gave rise to the exceptional Tribolium retinal mosaic.

\section{Tribolium LW- and UV-opsin expression is consistent with loss of B-opsin}

The expression of the Tribolium LW- and UV-opsins accounts for all photoreceptors in the Tribolium retina, and is compatible with a loss of B-opsin in the flour beetle. This finding further cements evidence for color vision reduction in the evolutionary lineage leading to Tribolium (Fig. 3). Consistent with this, the spectral sensitivity of the very closely related mealworm Tenebrio molitor has been reported to peak only in the UV and green wavelength ranges based on electroretinogram measurements [21]. Moreover, preliminary PCR screens from Tenebrio molitor genomic DNA resulted in isolation of closely related orthologs of Tribolium castaneum LW- and UV-opsins, while similar attempts to isolate B-opsin orthologs failed, just as in Tribolium castaneum (Bao and Friedrich, unpublished). Hence genome, gene expression and physiologi- cal data converge on supporting the loss of B-opsin in Tribolium castaneum.

\section{Loss of B-opsin expressing photoreceptors through expansion of the LW-opsin expression domain: cis- regulatory implications and possible trajectories}

Our data reveal that the lack of B-opsin in Tribolium correlates with a dramatic expansion of LW-opsin expression. In most endopterygote retinas studied so far, LW-opsins are expressed in the peripheral photoreceptors R1-6 and a fraction of central R7 or R8 cells (Fig. 3). Thus, the expanded LW-opsin we observe in Tribolium is likely to have played an essential role in the evolution of its retinal mosaic. The comparative data further imply that these changes involved the expansion of the peripheral LWopsin expression domain into R7 and R8.

A most accurate inference of the underlying mutational events underlying the Tribolium opsin expression pattern depends on knowing the organization of the retinal mosaic in the last B-opsin expressing ancestor of Tribolium. Although this is still much a matter of speculation, some conclusions can be drawn from physiological data from other Coleoptera and opsin expression data in other endopterygote orders. Based on electrophysiological studies, for instance, species in the beetle families Carabidae (ground beetles) and Coccinelidae (lady beetles) are equipped with sensitivities in the green, blue and UV $[22,23]$. Considering further that B-, LW- and UV-opsins are expressed in R7 and R8 cells in Hymenoptera, Lepidoptera and Diptera, it is most likely that Tribolium evolved from an ancestor in which R8 and R7 cells differentially expressed combinations of at least three opsins, similar to Drosophila (Fig. 3) [5,11,24].

Based on this premise, it is reasonable to assume that the UV-opsin expression in R7 is a conserved aspect of the Tribolium retina, whereas the loss of B-opsin expression and correlated expansion of LW-opsin expression is derived. This latter change may have occurred through one of several possible trajectories (Fig. 4). First, the loss of B-opsin expression could have been initiated through mutations that abrogate transcriptional activation or expression of a functional protein prior to or independent of changes in the ancestral expression domain of LW-opsin (Fig. 4a). However, this would create a retinal mosaic with partially light-insensitive photoreceptor cells. Considering the importance of flight in Tribolium (see below), the ensuing deterioration of spatial vision would likely be a devastating fitness penalty. This suggests that the molecular evolution of B-opsin loss was correlated with, or contingent upon, compensatory changes in the regulation of LWopsin, a second possibility (Fig. 4b). 


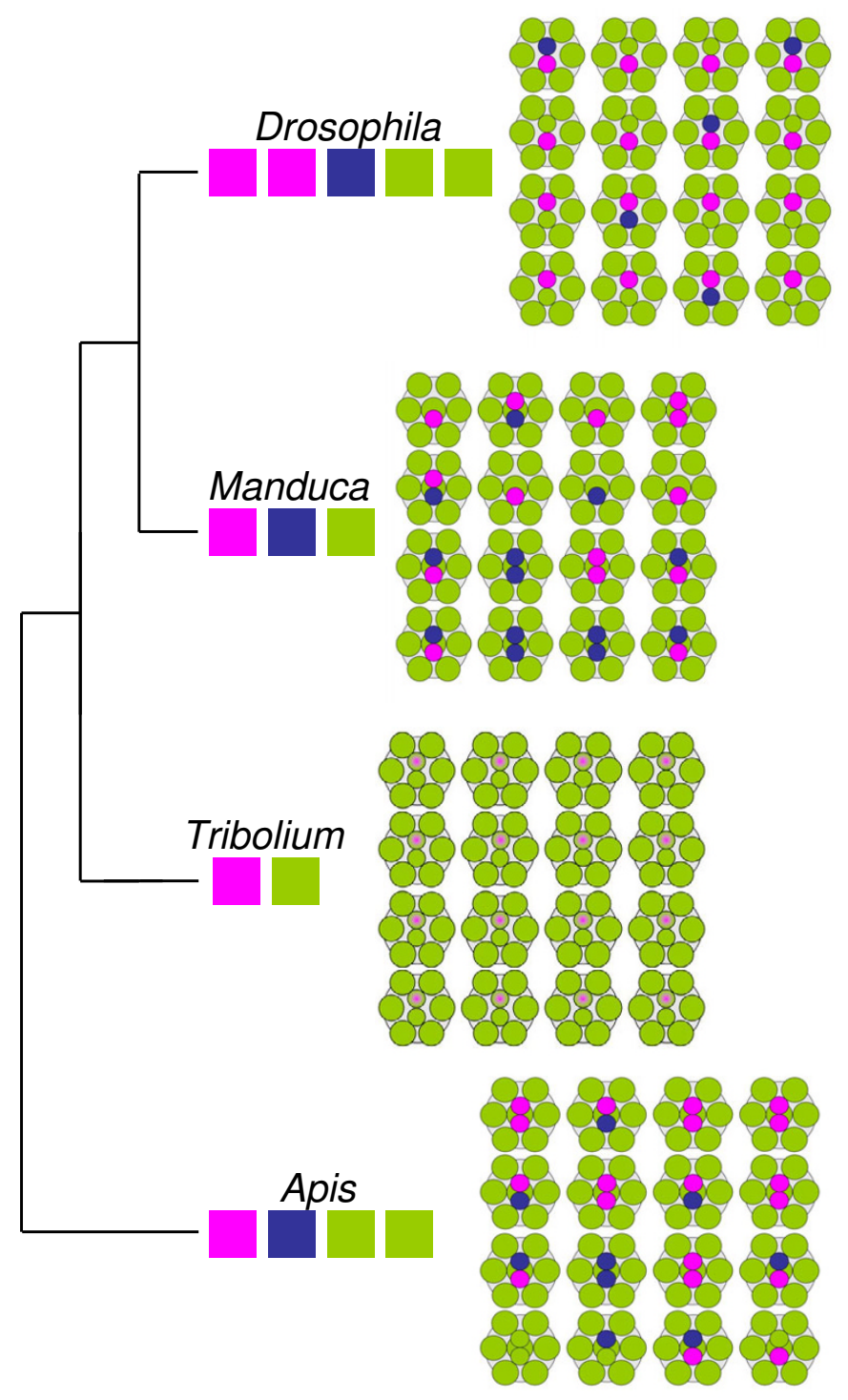

\section{Figure 3}

Comparison of insect photoreceptor mosaics. Schematic presentation of photoreceptor sensitivity arrays in the compound eyes of honeybee (Apis), tobacco hornworm moth (Manduca), Drosophila, and Tribolium. Colored boxes underneath genus names indicate number and wavelength specificities of opsins that are known to be expressed in the main retina. Tree visualizes phylogenetic relationships between the insect orders to which the four species belong [5I]. In the retinal mosaics, colors indicate photoreceptor light specificities in either the UV (violet), blue or green wavelength range. Drosophila forms eight photoreceptors per ommatidium. The peripheral photoreceptors RI-6 express a LW-opsin. 70\% of the Drosophila ommatidia are of the yellow type, in which the central R8 cell express a the LW-opsin paralog Rh6 and the central R7 cells the UV-opsin Rh4. In the remaining pale-type ommatidia, the central R8 cell express the B-opsin Rh5 and the central R7 cells the UV-opsin Rh3 (Fig. I) [12]. Honeybee and tobacco hornworm moth develop nine photoreceptors per ommatium due to duplication of the R7 cell fate [52]. However, in both species only the two central R7-like more distally located cells exhibit differential opsin expression ranging from UV to LW sensitive opsins. The peripheral photoreceptor cell homologs RI-6 express LW-opsin as does the proximally located central R8 cell homolog. Five different ommatidia types can be distinguished in the tobacco hornworm moth retina, which differ by number of R7-like cells (I-2) or the combination of B- and UV-opsin expressing R-7 cells [12]. In the honeybee, six different ommatidia types occur which either express G-, UV- or B-opsin in both R7-like cells or in any possible combination [24]. In Tribolium, LW-opsin is expressed in all photoreceptor cells. Co-expression of LW- with UV-opsin in R7 is indicated by gradient from violet to green. 
a.

Instantaneous B-opsin loss

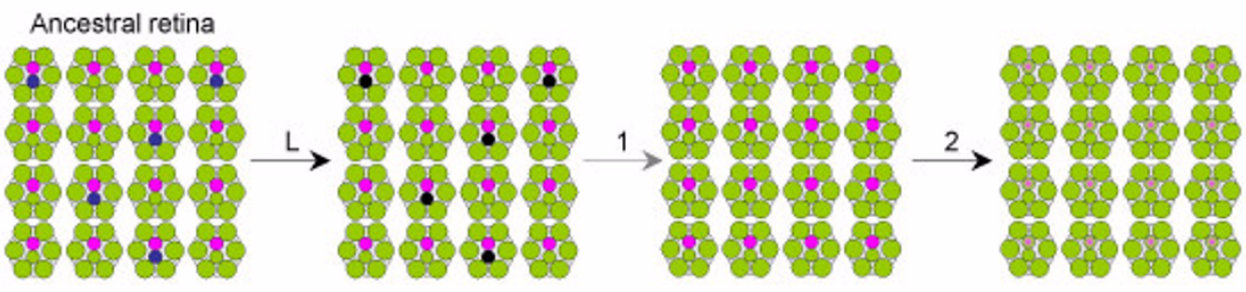

b.

Correlated LW-opsin domain expansion and B-opsin domain reduction

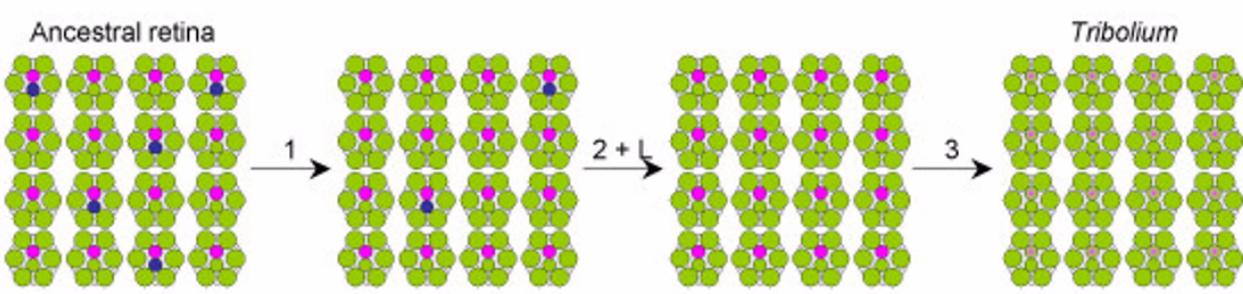

c. Instantaneous maximal LW-opsin domain expansion

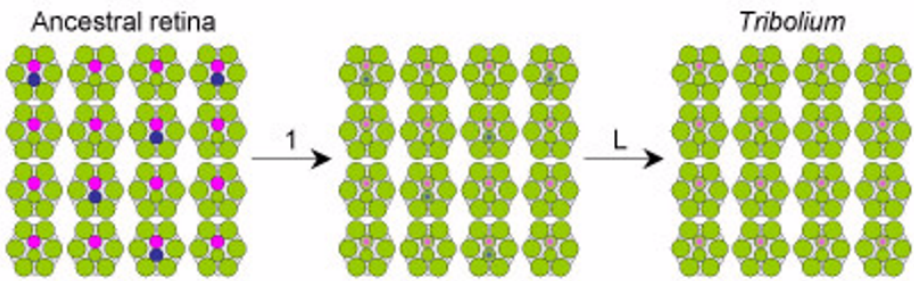

\section{Figure 4}

Possible trajectories of Tribolium retina evolution. Schematic presentation of three mutation scenarios that could have led from an ancestral trichromatic retina to the retinal mosaic of extant Tribolium castaneum. Colors indicate photoreceptor light specificities as in Fig. 3. Numbers above arrows represent number of necessary mutation induced gene regulatory changes underlying opsin expression pattern evolution. L stands for genomic loss of B-opsin. (a) Instantaneous B-opsin loss scenario. Bopsin loss without correlated or preceding expansion of other opsin expression domains is unlikely since it would result in a fraction of light insensitive photoreceptor cells and thereby a substantial deterioration of the resolution of spatial vision (black). Subsequent mutational steps that would lead to the Tribolium castaneum retinal mosaic are therefore just indicated with increased opacity. Genomic B-opsin loss was more likely preceded by either gradual expansion of the LW-opsin expression domain (b) or immediate maximum expansion of LW-opsin expression (c). See text for further details.

Several mechanisms exist regarding the nature of such cisregulatory changes in the B- and LW-opsin genes. Assuming heterogeneity of LW-opsin and B-opsin expression among ancestral R8 cells, for instance, correlated mutations in the cis-regulatory regions of both genes may have led to a gradual increase in the fraction of LW-opsin expressing cells that eventually culminated in the complete replacement of B-opsin expressing cells. This scenario describes a model of gradual replacement of blue versus green-sensitive photoreceptors in which color vision evolves as a quantitative trait (Fig. 4b). Alternatively, cis-regulatory change may have resulted in immedi- ate compensatory loss of B-opsin and expansion of LWopsin expression. This type of change could have been induced by separate mutations in the cis-regulatory regions of both genes or, as data from Drosophila suggest (see below), through mutational change in the cis-regulation of only one opsin pending pre-existing mechanisms of mutual repression. Both trajectories require at least one additional regulatory change that would allow further expansion of LW-opsin expression into R7 (Fig. 4b).

A third scenario is that a single mutation led to the immediate expansion of the LW-opsin expression into all pho- 
toreceptor cells. The consequence would be that of coexpression of LW-opsin with B-opsin in a fraction of R8 cells, and with UV-opsin in R7 cells (Fig. 4c). Such a retinal mosaic would thus be one step away from that in extant Tribolium by mutational disintegration of the activating elements in the ancestral B-opsin cis-regulatory region. This pathway seems the most probable for three reasons: (1) requiring only a single mutation to generate the final LW-opsin pattern is most parsimonious, (2) the co-expression of LW- and UV opsins in the R7 cells of Tribolium is consistent with this scenario, and (3) the latter fact as well as data from other species (see below) demonstrates that opsin co-expressing photoreceptors are viable and functional. Moreover, a strikingly similar scenario exists in Drosophila, where a single point mutation in the LW-opsin promoter, Rh6, or in the transcription factor, Orthodenticle (Otd) leads to expansion of Rh6 (and coexpression of opsins) into the majority of peripheral photoreceptors and many R7 central photoreceptors, but not into the B-opsin-containing R8 cells [25].

\section{Possible mechanisms for $L W$-opsin expansion: lessons from Drosophila}

Molecular genetic analyses in Drosophila have unraveled several critical mechanisms of cis-regulatory control of opsin expression, some of which are likely conserved $[5,12]$. Those particularly relevant to the discussion of the evolution of the Tribolium retina involve studies on the regulation of the LW-opsin-encoding gene, Rh6. Such studies have demonstrated that at least four independent transcriptional repression processes normally restrict Drosophila Rh6 to 70\% central R8 photoreceptors. In R8 cells, the mutually exclusive B- versus LW-opsin expression in 30 vs $70 \%$ R8 cells occurs through a bistable loop of both cell signaling and transcriptional regulation [26]. Failure to properly initiate this loop leads to a retina that expresses all B-opsin or all LW-opsin genes in R8 cells. Interestingly, LW-opsin expression is the "default" R8 opsin state, whereas B-opsin requires induction. Thus, this regulatory mechanism could reflect that the loss of $\mathrm{B}$ opsin observed in Tribolium is secondary to a loss in this induction process. This regulatory mechanism also suggests an inherent capacity of the photoreceptor mosaic in higher flies to evolve along the gradual replacement model considered for Tribolium above. Second, Drosophila express two LW-opsins: Rh1 in peripheral non-color sensitive photoreceptors, R1-R6, and Rh6 in 70\% R8 cells. Co-expression of both LW-opsins is specifically prevented in peripheral photoreceptors due to cell-specific repression of Rh6 [25]. A third mechanism of repression for Rh6 occurs in all R7 cells, where it is repressed by the R7-specific transcription factor, Prospero (Pros) [27]. Finally, $R h 6$ expression in DRA ommatidia (both R7 and R8 cells) is prevented through the selective inactivation of the R8specific, Rh6 activating transcription factor Senseless
(Sens) in the DRA [28]. Thus, the release of analogous LW-opsin repression mechanisms could have led to the expansion of LW-opsin expression as postulated for the evolution of Tribolium.

While it is premature to draw final conclusions on the trajectory of cis-regulatory changes that generated the retinal mosaic of Tribolium castaneum, data and implications identify Tribolium as unique model to study compensatory gene regulatory mechanisms associated with gene loss. Progress in this area can be made by studying the cis-regulatory control of Tribolium LW-opsin in comparison to that of Drosophila Rh6 and Rh1, and by exploring the conservation of opsin expression in a wider range of darkling beetles, as well as in distantly related Coleoptera.

\section{Consequences and significance of opsin co-expression in the Tribolium $R 7$ cells}

Across orders, the "one receptor rule" is important for sensory receptor expression $[3,29]$. The only two exceptions to this rule in insect photoreceptors reported thus far occur in butterfly species that have regionalized photoreceptors that co-express two opsins [30,31]. Thus, Tribolium is the first insect example in which a specific photoreceptor subtype co-expresses two different opsins across the entire retina. Opsin co-expression is more common in vertebrates than invertebrates [32]. In the Siberian hamster and the pouched mouse, for instance, dual opsin-expressing cone cells are uniformly spread throughout the retina similar to Tribolium [33]. Intriguingly, vertebrate species with exceptionally high frequencies of opsin co-expressing photoreceptors are either nocturnal or fossorial $[32,34]$. This evolutionary parallel raises the possibility that opsin co-expression is of advantage under conditions where brightness sensitivity is critical. This bodes well with the low light ecological niche of Tribolium since all postembryonic stages of Tribolium show strong negative phototaxis $[35,36]$.

Both vertebrate and insect opsin co-expressing photoreceptors have been found sensitive to a light spectrum that equals the summation of the single sensitivities of the expressed opsins $[30,37,38]$. While the excitation characteristics of the Tribolium R7 photoreceptors await to be analyzed by electrophysiological means, it is reasonable to assume that these cells are sensitive to a broad range of light from the green into UV wavelengths. What then may have caused the evolution of this unusual trait? For this question to be answered, it is necessary to further explore the functional consequences of opsin co-expression and, secondly, the state from which the Tribolium patterns originated.

Modeling data suggest that optimal single opsin-containing photoreceptors exhibit higher photon capture rate 
than dual opsin-expressing photoreceptors [6]. However, physiological measurements and ecological correlations point to a stronger impact of extended wavelength sensitivity for enhancing brightness detection. In house fly, for instance, the LW-opsin-expressing peripheral photoreceptors R1-6 contain a UV sensitizing pigment [39], which leads to an expanded wavelength sensitivity similar to that assumed for the Tribolium R7 cells. Importantly, this supplemental UV-sensitizing pigment has been shown to significantly increase overall brightness sensitivity in the peripheral photoreceptors of the house fly. The effect is considered to be particularly significant under low light conditions [39]. Interestingly, Tribolium castaneum does disperse by flight under conditions of stress or exposure to strong light [40]. The conservation of flight capability indicates that flight has maintained fitness importance throughout Tribolium evolution. Moreover, adults of the meal worm Tenebrio molitor are negatively phototactic like Tribolium but become positively phototactic after extended times of desiccation [41], suggesting that this closely related species to Tribolium behaves as a facultative cryptozoic species. Thus, the Tribolium visual system may have been molded by long-term cryptozoic adaptation.

From which functional ancestral state did the broadband sensitivity of Tribolium R7 cells evolve? While the function of the Tribolium R7 photoreceptors may have changed to extreme broadband photoreceptors to boost the overall photon capture capacity of the retina, enhanced brightness sensitivity is not incompatible with maintenance of color detection capacity. In theory, excitation differences between LW-opsin expressing R8 and LW/UV-opsin-coexpressing R7 cells may still discriminate UV from green patterns to facilitate dichromatic vision in Tribolium. The former may have evolved without completely sacrificing UV versus LW discrimination ability. Indeed, structural and comparative evidence suggest that the Tribolium central photoreceptor cells R7 and R8 represent ancestral color detectors. The cellular architecture of the Tribolium ommatidia is very similar to that in mealworm Tenebrio molitor. In both species, the six peripheral photoreceptors form a ring of rhabdomeres while the inner R7 and R8 photoreceptors contribute a separate rhabdom stem $[13,42]$. Importantly, Tenebrio ommatidia exercise pupillike brightness adaptation through volume changes of the primary pigment cells which sit on top of the photoreceptor bundle [42]. In the light-adapted state, the primary pigment cells are expanded such that only the central R7 and $\mathrm{R} 8$ cells are exposed to the light path. In the darkadapted state, the primary pigment cells further contract to additionally expose the peripheral R1-6 to light. This mechanism suggests specialization or division of brightness detection versus color discrimination tasks between inner and outer photoreceptors, similar to Drosophila. While this interpretation remains to be experimentally tested, the fact that the Tenebrio light adaptation mechanism is conserved in the more distantly related darkling species Zophobas atratus strongly suggests that the Tribolium situation evolved from a central photoreceptor-based color discriminating system [42].

Taken together, there is circumstantial evidence for both costs and benefits to opsin co-expression in Tribolium R7 cells. Thus, it will be necessary to elucidate the relative significance of these aspects for the biology of Tribolium to understand the evolutionary forces that generated the Tribolium retinal mosaic. Importantly, while dispersal by flight under conditions of stress seems to represent the likeliest condition under which brightness sensitivity may be critical for Tribolium, observations in the laboratory suggest little dependence of food and mating partner detection on vision [35]. Nonetheless, the cryptozoic habits of Tribolium speak for an increased selection pressure on brightness sensitivity and relaxation of the selection pressure on color discrimination needs.

\section{Neutral or adaptive replacement of B-opsin?}

The most parsimonious trajectory of cis-regulatory evolution that leads to the retinal mosaic of extant Tribolium is mechanistically linked to both the emergence of UV-/LWopsin co-expressing R7 cells and the loss of B-opsin expression in R8 cells (Fig. 4c). The potential adaptive advantage of UV-/LW-opsin co-expression in Tribolium R7 cells generates the question: could the loss of B-opsin have been influenced not only by relaxation of color vision needs but also enforced by an adaptive effect of increased brightness sensitivity? Lack of blue sensitivity is reliably documented in only a few cases of insects, including the crepuscular cockroach Periplaneta americana, and the owlfly Ascalaphus macarionius, a diurnal predator $[43,44]$. The diverse behavioral patterns of these species, however, have provided little information regarding the potential causes leading to reduced color discrimination [2]. Remarkably, in vertebrates, opsin dual-expressing photoreceptors as well as single LW-sensitive photoreceptors are increased in species with cryptozoic life histories (for review see [34]). Keeping in mind that brightness detection is primarily based on LW-opsins in both vertebrates and insects, the parallel increase of LW-opsin expressing cells in cryptozoic vertebrates and Tribolium is further suggestive of an adaptive trend. The replacement of B-opsin with LW-opsin expressing cells during Tribolium evolution may thus have been adaptive. However, this does not enforce the conclusion that the loss of Tribolium B-opsin involved positive selection. Either gradual or complete expansion of the LW-opsin followed by decay of B-opsin expression may have occurred as neutral or permissively deleterious changes in color vision lost fitness importance, while brightness sensitivity remained under purifying selection in the lineage leading to Tribolium. It is not 
clear whether the similarity in the opsin repertoires of cryptozoic insect or vertebrate retinas reflects convergent preferential conservation of photoreceptors essential for brightness detection or adaptive enrichment within a short time window of cryptozoic adaptation [34]. Extended comparative data may provide further insights. For instance, conservation of specific UV-opsin expression but not LW- and UV-opsin co-expression in R7 cells in related darkling beetles would indicate a persisting need of detecting UV light patterns but point against a strong advantage of brightness sensitivity increase through LWand UV co-expression. Conserved co-expression of UV with LW-opsin in R7, on the other hand, would be most reasonably correlated with a fitness advantage of maximizing brightness sensitivity.

\section{Conclusion}

With many questions awaiting to be addressed, it seems clear that the first look into the retinal mosaic of a cryptozoic coleopteran marks just the entry point for studying the dark side of insect vision. Besides serving as a model for development and pest biology, which provided the rationale for genome sequencing [45], Tribolium also holds promise to add illuminating data to the classic debate over the mechanisms of reductive phenotype evolution in cryptozoic animals [46].

\section{Methods \\ Animal stocks}

Pearl pBac(3xP3-EGFP)af T. castaneum were reared on 5\% yeast supplemented whole wheat flour at $31^{\circ} \mathrm{C}$ in constant darkness. $y^{1} w^{67}$ Drosophila melanogaster lines were maintained on standard cornmeal-molasses food at room temperature.

\section{Bioinformatics}

The putative coding regions of Tribolium castaneum LWopsin and UV-opsin were identified by TBLASTX search with the Drosophila Rh1 and Rh3 gene sequences to the Tribolium castaneum Georgia GA2 genome assembly (release 8/17/2005), accessed through the Human Genome Sequencing Center server in the Department of Genetics at Baylor University [47]. Similar searches with the remaining Drosophila opsins, Rh2, Rh4-Rh6, failed to identify additional Tribolium opsin-encoding genes.

\section{Molecular biology}

A 502 bp fragment of the predicted Tribolium UV-opsin gene was amplified from Tribolium total genomic DNA by standard PCR using the specific primers 5'-CCCCAAAGACGAACTCATTC-3' and 5'-ATGGTGTACCCCCAAACAAA-3'. This primer combination produces a PCR amplicon that extends from the first putative open reading frame (ORF) containing exon of the Tribolium UVopsin gene to the second ORF-containing exon bridging a
$55 \mathrm{bp}$ intron. $972 \mathrm{bp}$ of the predicted Tribolium LW-opsin ORF were amplified by nested RT-PCR using outer primer pair TcRhR-3F (5'-TCACCGTCGTGGACAAAGTC-3') and TcRhR-3R (5'-TTCTCGTCGGTGGTAACGTTC-3'), and inner primer pair TcRhR-4F (5'-GCCCATTGGTACCAGTTCC-3') and TcRhR-4R (5'-AGCCGTGGAGGTGGTGTC3'). Total RNA was extracted from adult Tribolium head with the RNAqueous kit (Ambion). The RETROscript kit (Ambion) was used to generate cDNA through reverse transcription initiated with random decamers. The resulting PCR fragments were cloned into pGEM $^{\circledR}$-T Vector (Promega). Multiple identical sequences were obtained for each paralog. The sequences are accessible under accession numbers [GenBank: DQ029113] and [GenBank: EU164547].

\section{In situ hybridization, immunohistochemistry, and microscopy}

95\% pupal Drosophila whole-mounted retinas were dissected at room temperature (RT) in PBT $(1 \times \mathrm{PBS}+0.1 \%$ Triton X100, pH 7.2), fixed in PLP ( $1 \times$ PBS, 4\% paraformaldehyde, $0.075 \mathrm{M}$ lysine, $0.01 \mathrm{M}$ sodium periodate, $0.05 \%$ saponin) [48], and washed $3 \times 10 \mathrm{~min}$ with PBT. Retinas were transferred to Signal-iT FX (Invitrogen) for $30 \mathrm{~min}, \mathrm{RT}$, before incubation with mouse anti-Rh1 (1:100, DHSB), mouse anti-Rh3 (1:5, gift from S.G. Britt), and rabbit anti-Rh4 (1:250, gift from C.S. Zuker) overnight at $4^{\circ} \mathrm{C}$ in BNTS $(1 \times$ PBT, $1.5 \mathrm{M} \mathrm{NaCl}, 0.1 \%$ BSA, $0.05 \%$ saponin). Samples were washed $3 \times 20 \mathrm{~min}$ with PBT, incubated for 2 hrs at RT with AlexaFluor-488, -555, or -648-conjugated secondary antibodies (1:500, Molecular Probes), and washed thrice again with PBT. Retinas were mounted in Prolong Gold antifade-reagent (Invitrogen), and imaged $24 \mathrm{hr}$ later using an Axioplan Ilie equipped with the Zeiss ApoTome deconvolution module. Digital images were processed with Axiovision 4.5 (Zeiss) and AdobePhotoshop 7.0 software.

Whole mount in situ hybridization of Tribolium pupal retinae was carried out following previously published protocols with minor modifications [49,50]. Digoxygeninlabeled antisense RNA probes were prepared with DIGUTP RNA Labeling Mix (Roche). Pupal retinas were dissected in ice cold PBS, and fixed in $9.25 \%$ formaldehyde, $50 \mathrm{mM}$ EGTA ph8, and 10\% Triton X in PBT at RT for $1 \mathrm{~h}$. Tissues were then washed one time in PBS, three times in PBT, and equilibrated for hybridization with a $10 \mathrm{~min}$ incubation in 50\% PBT+50\% Hyb-A-RNA (hybridization buffer A) at RT, and $1 \mathrm{~h}$ at $55^{\circ} \mathrm{C}$ in $100 \%$ Hyb-A-RNA (50\% formamide, $4 \times$ SSC, $250 \mu \mathrm{g} / \mathrm{ml}$ tRNA, $0.1 \%$ Tween20, $1 \times$ Denhardt's solution, 5\% dextran sulfate) with 500 $\mu \mathrm{g} / \mathrm{ml}$ freshly denatured herring sperm DNA. Hybridization was carried out for $72 \mathrm{~h}$ in Hyb-A-RNA with RNA probe at approximately $0.5 \mu \mathrm{g} / \mathrm{ml}$ concentration at $55^{\circ} \mathrm{C}$. Subsequently, pupal retinas were washed $3 \times 30 \mathrm{~min}$ in 
Hyb-B-RNA (50\% formamide, $4 \times$ SSC, $0.1 \%$ Tween-20) at $55^{\circ} \mathrm{C}$ followed by a $24 \mathrm{hrs}$ final washing step. Next, tissues were equilibrated in $5 \times$ SSC at RT for $5 \mathrm{~min}$ and another $5 \mathrm{~min}$ in maleic acid buffer $(0.1 \mathrm{M}$ maleic acid, $0.15 \mathrm{M} \mathrm{NaCl}, 0.1 \%$ Tween-20). Tissue was blocked for 2 hrs at RT in maleic acid buffer supplemented with Roche in situ blocking buffer at 1:10 dilution (Roche). Anti-DIG antibody (Roche) was added at a 1:5000 dilution and tissues were incubated 2 hrs at RT on a shaker. Following antibody incubation, pupal eye discs were washed $3 \times 1$ hrs. Tissues were then transferred into detection buffer (0.1 M Tris-HCl pH 9.5, 0.1 M NaCl, $50 \mathrm{mM} \mathrm{MgCl}_{2}$ ) for 5 min at RT. Color detection was initiated by adding $4.5 \mu \mathrm{l}$ NBT $(50 \mathrm{mg} / \mathrm{ml})$ and $3.5 \mu \mathrm{lBCIP}(50 \mathrm{mg} / \mathrm{ml})$ as substrate. Color reactions were developed for 1 to $2 \mathrm{hrs}$ and stopped by replacing staining buffer with PBT. Labeled retinas were washed with EtOH, rehydrated, and cleared in 70\% glycerol in PBS before mounting. DIC brightfield images were recorded through a Zeiss Axioscop coupled to a SPORT RT camera (Diagnostic Instruments). Contrast and brightness were adjusted in Photoshop CS3.

\section{Competing interests}

The author(s) declare that they have no competing interests.

\section{Authors' contributions}

MJ carried out Tribolium LW- and UV-opsin expression analysis, RB cloned the Tribolium UV-opsin gene fragment and carried out gene ortholog searches, ZL cloned the Tribolium LW-opsin RT-PCR fragment, ECM generated Drosophila opsin expression data, TAC designed analysis of Drosophila opsin expression and co-wrote the manuscript, MF conceived the study and wrote the manuscript.

\section{Acknowledgements}

We are grateful to Elke K. Buschbeck, and Karen L. Myhr for valuable comments on the manuscript, to Charles S. Zuker, Steve G. Britt, and the Developmental Studies Hybridoma Bank developed under the auspices of the NICHD and maintained by the University of lowa for antibodies, Dick W. Beeman for sharing ortholog search results with unpublished EST data, and two anonymous reviewers for thorough and helpful comments. TAC was supported by Cincinnati Children's Hospital Medical Center, Research Preventing Blindness and the E. Matilda Ziegler Foundation for the Blind, and ECM was supported by Ohio Preventing Blindness and NIH T32 HD046387.

\section{References}

I. Hardie RC, Raghu P: Visual transduction in Drosophila. Nature 200I, 413(6852): 186-193.

2. Briscoe $A D$, Chittka $L$ : The evolution of color vision in insects. Annu Rev Entomol 200I, 46:47I-5I0.

3. Kelber A: Invertebrate colour vision. In Invertebrate vision Edited by: Warrant E, Nilsson DE. New York , Cambridge University Press; 2006:250-290.

4. Stavenga DG, Arikawa K: Evolution of color and vision of butterflies. Arthropod Structure \& Development 2006, 35:307-3 I8.

5. Morante J, Desplan C, Celik A: Generating patterned arrays of photoreceptors. Curr Opin Genet Dev 2007, I 7(4):3 I4-3I9.
6. Osorio D, Vorobyev M: Photoreceptor spectral sensitivities in terrestrial animals: adaptations for luminance and colour vision. Proceedings of the Royal Society B-Biological Sciences 2005, 272(1574): I745-I752.

7. Velarde RA, Sauer CD, Walden KK, Fahrbach SE, Robertson HM: Pteropsin: a vertebrate-like non-visual opsin expressed in the honey bee brain. Insect Biochem Mol Biol 2005, 35(12): 1367-1377.

8. Tribolium-Genome-Sequencing-Consortium: The genome of the developmental model and pest Tribolium castaneum. in review 2007.

9. Dendy A: The cryptozoic fauna of Australia. Rept Australian Assoc Adv Sci 1895, 6:99-I 19.

10. Sokoloff A: The biology of Tribolium. Volume I. Oxford, Clarendon Press; 1972.

II. White $\mathrm{RH}, \mathrm{Xu} \mathrm{H}$, Munch TA, Bennett RR, Grable EA: The retina of Manduca sexta: rhodopsin expression, the mosaic of green-, blue- and UV-sensitive photoreceptors, and regional specialization. J Exp Biol 2003, 206(Pt I 9):3337-3348.

12. Cook T, Desplan C: Photoreceptor subtype specification: from flies to humans. Semin Cell Dev Biol 200I, I 2(6):509-5 I8.

13. Friedrich M, Rambold I, Melzer RR: The early stages of ommatidial development in the flour beetle Tribolium castaneum (Coleoptera, Tenebrionidae). Dev Genes Evol 1996, 206: I 36-I 46.

14. Earl JB, Britt SG: Expression of Drosophila rhodopsins during photoreceptor cell differentiation: insights into R7 and R8 cell subtype commitment. Gene Expr Patterns 2006, 6(7):687-694.

15. Montell C, Jones K, Zuker C, Rubin G: A second opsin gene expressed in the ultraviolet-sensitive $R 7$ photoreceptor cells of Drosophila melanogaster. J Neurosci 1987, 7(5):| 558-I566.

16. Huber A, Schulz S, Bentrop J, Groell C, Wolfrum U, Paulsen R: Molecular cloning of Drosophila Rh6 rhodopsin - the visual pigment of a subset of R8 photoreceptor cells. Febs Letters 1997, 406:6-10.

17. Chou WH, Hall KJ, Wilson DB, Wideman CL, Townson SM, Chadwell LV, Britt SG: Identification of a novel Drosophila opsin reveals specific patterning of the $\mathrm{R7}$ and $\mathrm{R} 8$ photoreceptor cells. Neuron 1996, I7(6): I101-III5.

18. Papatsenko D, Sheng G, Desplan C: A new rhodopsin in R8 photoreceptors of Drosophila: evidence for coordinate expression with Rh3 in R7 cells. Development 1997, I 24(9): | 665- I673.

19. Fortini ME, Rubin GM: Analysis of cis-acting requirements of the Rh3 and Rh4 genes reveals a bipartite organization to rhodopsin promoters in Drosophila melanogaster. Genes Dev 1990, 4(3):444-463.

20. Labhart T, Meyer EP: Detectors for polarized skylight in insects: a survey of ommatidial specializations in the dorsal rim area of the compound eye. Microsc Res Tech 1999, 47(6):368-379.

21. Yinon U: The visual mechanisms of Tenebrio molitor: some aspects of the spectral response. The Journal of Experimental biology 1970, 53(I):221-229.

22. Hasselmann EM: Ueber die relative spektrale Empfindlichkeit von Kaefer- und Schmetterlingsaugen bei verschiedenen Helligkeiten. Zool Jb Physiol 1962, 69:537-576.

23. Lin JT: Identification of photoreceptor locations in the compound eye of Coccinella septempunctata Linnaeus (Coleoptera). Journal of Insect Physiology 1993, 39(7):555-562.

24. Wakakuwa M, Kurasawa M, Giurfa M, Arikawa K: Spectral heterogeneity of honeybee ommatidia. Naturwissenschaften 2005, 92(1 0):464-467.

25. Tahayato A, Sonneville R, Pichaud F, Wernet MF, Papatsenko D, Beaufils $P$, Cook T, Desplan C: Otd/Crx, a dual regulator for the specification of ommatidia subtypes in the Drosophila retina. Dev Cell 2003, 5(3):391-402.

26. Mikeladze-Dvali T, Wernet MF, Pistillo D, Mazzoni EO, Teleman AA, Chen YW, Cohen S, Desplan C: The growth regulators warts/ lats and melted interact in a bistable loop to specify opposite fates in Drosophila R8 photoreceptors. Cell 2005, I 22(5):775-787.

27. Cook T, Pichaud F, Sonneville R, Papatsenko D, Desplan C: Distinction between color photoreceptor cell fates is controlled by Prospero in Drosophila. Dev Cell 2003, 4(6):853-864.

28. Xie B, Charlton-Perkins M, McDonald EC, Gebelein B, Cook T: Senseless functions as a molecular switch for color photore- 
ceptor differentiation in Drosophila. Development 2007, 134(23):4243-4253.

29. Mazzoni EO, Desplan C, Celik A: 'One receptor' rules in sensory neurons. Dev Neurosci 2004, 26(5-6):388-395.

30. Arikawa K, Mizuno S, Kinoshita M, Stavenga DG: Coexpression of two visual pigments in a photoreceptor causes an abnormally broad spectral sensitivity in the eye of the butterfly Papilio xuthus. J Neurosci 2003, 23(I I):4527-4532.

31. Sison-Mangus MP, Bernard GD, Lampel J, Briscoe AD: Beauty in the eye of the beholder: the two blue opsins of lycaenid butterflies and the opsin gene-driven evolution of sexually dimorphic eyes. J Exp Biol 2006, 209(Pt I6):3079-3090.

32. Lukats A, Szabo A, Röhlich P, Vigh B, Szel A: Photopigment coexpression in mammals: comparative and developmental aspects. Histology and Histopathology 2005, 20(2):55I-574.

33. Lukats A, Dkhissi-Benyahya O, Szepessy Z, Röhlich P, Vigh B, Bennett NC, Cooper HM, Szel A: Visual pigment coexpression in all cones of two rodents, the Siberian hamster, and the pouched mouse. Investigative Ophthalmology \& Visual Science 2002, 43(7):2468-2473.

34. Peichl L: Diversity of mammalian photoreceptor properties: Adaptations to habitat and lifestyle? The Anatomical Record Part a - Discoveries in Molecular Cellular and Evolutionary Biology 2005, 287A(I): $|00|-1012$.

35. Park T: Observations on the general biology of the flour beetle, Tribolium confusum. Quart Rev Biol 1934, 9:36-64.

36. Soderstrom EL: Phototactic response of adults of confused flour beetle and red flour beetle and larvae of black carpet beetle to flashes of blue-white light. J econ Ent 1968:973-975.

37. Feiler R, Bjornson R, Kirschfeld K, Mismer D, Rubin GM, Smith DP, Socolich M, Zuker CS: Ectopic expression of ultraviolet-rhodopsins in the blue photoreceptor cells of Drosophila: visual physiology and photochemistry of transgenic animals. J Neurosci 1992, I 2(10):3862-3868.

38. Lyubarsky AL, Falsini B, Pennesi ME, Valentini P, Pugh EN Jr.: UV- and midwave-sensitive cone-driven retinal responses of the mouse: a possible phenotype for coexpression of cone photopigments. J Neurosci 1999, 19(1):442-455.

39. Stavenga DG: Visual acuity of fly photoreceptors in natural conditions--dependence on UV sensitizing pigment and light-controlling pupil. J Exp Biol 2004, 207(Pt I 0): I703-1713.

40. Good NE: Biology of the flour beetles, Tribolium confusum Duv., and T.ferrugineum. J Agr Res 1933, 46:327-334.

4I. Perttunen $V$, Lahermaa $M$ : The light reactions of the larvae and adults of Tenebrio molitor $\mathrm{L}$. (Col., Tenebrionidae) and their interference with the humidity reactions. Ann ent Fenn 1963 , 29:83-106.

42. Ro Al, Nilsson DE: Sensitivity and dynamics of the pupil mechanism in two tenebrionid beetles. Journal of Comparative Physiology a-Sensory Neural and Behavioral Physiology 1993, I73(4):455-462.

43. Mote MI, Goldsmith TH: Spectral sensitivities of color receptors in the compound eye of the cockroach Periplaneta. J Exp Zool 1970, I73:137-45.

44. Gogala M: Die spektrale Empfindlichkeit der Doppelaugen von Ascalaphus macaronius Scop. (Neuroptera, Ascalaphidae). Z Vergl Physiol 1967, 57:232-43.

45. Brown SJ, Denell RE, Beeman RW: Beetling around the genome. Genet Res 2003, 82(3): 155-161.

46. Culver DC, Wilkens $\mathrm{H}$ : Critical review of the relevant theories of the evolution of subterranean animals. In Ecosystems of the world: subterranean animals Edited by: Wilkens H, Culver DC, Humphries WF. Amsterdam , Elsevier; 2000

47. Altschul SF, Madden TL, Schaffer AA, Zhang J, Zhang Z, Miller W, Lipman D]: Gapped BLAST and PSI-BLAST: a new generation of protein database search programs. Nucleic Acids Res 1997 25(I7):3389-3402.

48. McLean IW, Nakane PK: Periodate-lysine-paraformaldehyde fixative. A new fixation for immunoelectron microscopy. J Histochem Cytochem 1974, 22:1077-1083.

49. Broadus J, Doe CQ: Evolution of neuroblast identity: seven-up and prospero expression reveal homologous and divergent neuroblast fates in Drosophila and Schistocerca. Development 1995, I 2 I ( I 2):3989-3996.

50. Friedrich M, Benzer S: Divergent decapentaplegic expression patterns in compound eye development and the evolution of insect metamorphosis. Journal of Experimental Zoology (Molecular and Developmental Evolution) 2000, 288:39-55.

5I. Savard J, Tautz D, Richards S, Weinstock GM, Gibbs RA, Werren JH, Tettelin H, Lercher MJ: Phylogenomic analysis reveals bees and wasps (Hymenoptera) at the base of the radiation of Holometabolous insects. Genome Res 2006, I 6(I I): I334-I338.

52. Ready DF: A multifaceted approach to neural development. Trends In Neurosciences 1989, 12:102-I I0.
Publish with Biomed Central and every scientist can read your work free of charge

"BioMed Central will be the most significant development for disseminating the results of biomedical research in our lifetime. "

Sir Paul Nurse, Cancer Research UK

Your research papers will be:

- available free of charge to the entire biomedical community

- peer reviewed and published immediately upon acceptance

- cited in PubMed and archived on PubMed Central

- yours - you keep the copyright 